\title{
Genetic Association of MiR-146a with Multiple Sclerosis Susceptibility in the Chinese Population
}

\author{
You Lia Chen Du ${ }^{b}$ Wei Wang ${ }^{c}$ Guoda Ma ${ }^{a}$ Lili Cui ${ }^{a}$ Haihong Zhou ${ }^{d}$ \\ Hua Tao ${ }^{d}$ Lifen Yao ${ }^{c}$ Bin Zhao ${ }^{\text {a,d }}$ Keshen Lia \\ aGuangdong Key Laboratory of Age-Related Cardiac and Cerebral Diseases, Affiliated Hospital \\ of Guangdong Medical College, Zhanjiang, 'Harbin Medical University Cancer Hospital, Harbin, \\ 'Department of Neurology, The First Affiliated Hospital of Harbin Medical University, Harbin, \\ dDepartment of Neurology, Affiliated Hospital of Guangdong Medical College, Zhanjiang China
}

\section{Key Words}

MiR-146a • Multiple sclerosis • Polymorphism

\begin{abstract}
Background: miR-146a polymorphisms have been involved in susceptibility to multiple diseases. The aim of the present study was to analyze the potential association between two functional miR-146a polymorphisms (rs2910164 and rs57095329) and multiple sclerosis (MS) in the Han Chinese population. Methods: A cohort of 525 patients and 568 healthy controls were genotyped to detect the two polymorphisms by SNaPshot. Results: No significant differences were detected in the distribution of the two miR-146a polymorphisms between the patients and controls $(P>0.05)$. However, stratification by gender showed a statistically significant difference in the frequency of the genotype rs2910164 between MS patients and control females $(P=0.009)$. Further stratification analysis by subgroup revealed that the miR$146 a$ rs2910164 C allele conferred a higher risk of developing relapsing-remitting MS (RRMS) $(\mathrm{P}=0.018)$. In addition, the rs2910164 C allele was significantly associated with increased expression of miR-146a in patients with RRMS $(P=0.025)$. Moreover, patients with the rs2910164 C allele released more TNF- $\alpha$ and IFN- $\gamma$, but not IL-1 $\beta$, compared with individuals carrying the homozygous GG genotype $(P<0.05)$. Conclusions: Our results provide evidence that rs2910164 may play a role in MS susceptibility in females. The rs2910164 G>C variation may affect the expression of miR-146a and the release of proinflammatory cytokines.
\end{abstract}

Y. Li, C. Du and W. Wang equal contributors.

Copyright $\odot 2015$ S. Karger AG, Basel 
Li et al.: MiR-146a Polymorphisms and Multiple Sclerosis

\section{Introduction}

Multiple sclerosis (MS) is a chronic, inflammatory, demyelinating neurodegenerative disease of the central nervous system (CNS) afflicting two million people worldwide [1]. The disease is characterized by infiltration of the CNS with autoreactive immune cells, demyelination, acute astrogliosis, and axonal damage due to immune reactions against the myelin sheath [2]. Approximately $80 \%$ of MS patients diagnosed with relapsing-remitting MS (RRMS) develop a secondary, progressive MS course over the following decade. In a smaller group of patients (20\%), MS begins with a primary, progressive phase (PPMS) [3]. Although the precise molecular pathogenic mechanism of MS still remains elusive, there is solid evidence that both genetic and environmental factors are implicated in MS susceptibility [4]. The major histocompatibility complex on chromosome 6 p21.3 has been well studied, is thought to be strongly associated with MS susceptibility, and has been unambiguously identified in all studied populations [5]. A large body of scientific research indicates that the HLA-DRB1 gene is the main genetic factor for MS. However, the HLA (human leukocyte antigen) genes account for only half of the genetic liability to the disease [6]. Therefore, in recent years, multiple, non-HLA genes have been implicated in susceptibility to MS, but many other genes remain to be discovered.

MicroRNAs (miRNAs) represent a family of small ( 22 nucleotides), endogenous, noncoding RNAs that modulate the expression of multiple target mRNAs by inducing either translational inhibition or mRNA degradation. MiRNAs are critically involved in various physiological and pathological processes, including the regulation of innate and adaptive immune responses [7]. One of these conserved miRNAs is miR-146a, which is well known for its powerful regulation of immune response and inflammation. MiR-146a, located in the LOC285628 gene on human chromosome 5, is involved in modulating the negative regulation of Toll like receptor (TLR) signaling, airway remodeling-associated proteins (MMP-13 and collagen II), and pivotal inflammatory cytokines [8]. The dysregulation of miR-146a is associated with multiple autoimmune diseases, such as systemic lupus erythematosus (SLE) [9], rheumatoid arthritis (RA) [10], Sjogren's syndrome (SS) [11], and MS [12]. Recently, miR146a was found to be upregulated in active MS lesions [13] in humans and in experimental autoimmune encephalomyelitis (EAE) lesions of mice and marmosets [14], which suggests that miR-146a plays a pivotal role in the pathophysiology of MS.

Polymorphisms affecting miRNA expression, maturation, or mRNA recognition may represent an important risk determinant of disease susceptibility. The $\mathrm{C}$ allele of the rs2910164 G>C polymorphism of miR-146a causes mis-pairing within the miR-146a hairpin, decreased expression of its mature form, and abated expression of its target genes, including TNF receptor-associated factor 6 (TRAF6) and IL-1 receptor-associated kinase 1 (IRAK1) [15]. This functional polymorphism was most extensively studied for its association with various diseases, including temporal lobe epilepsy [16], asthma [17], SLE [9, 18], and RA [10]. A very recent study also demonstrated that the rs57095329 $A>G$ polymorphism in the promoter region of miR-146a was associated with SLE in Chinese individuals, and the mutant allele alleviated miR-146a expression through the reduction of its binding affinity for the transcription factor Ets-1 [18]. To date, there are few studies that have investigated the association between single miR-146a polymorphisms and MS susceptibility, and the only case-controlled study for miR-146a rs2910164 performed in Caucasians failed to find any differences in the frequencies of rs2910164 between patients and controls [19]. Given the critical regulatory role of miR-146a in immune response and its elevation in MS, it is likely that polymorphisms in the miR-146a gene could potentially confer disease risk. In the present study, we performed a case-controlled study to ascertain whether the two polymorphisms within the miR-146a gene are associated with MS susceptibility in the Chinese population and whether these polymorphisms are associated with miR-146a expression and proinflammatory cytokine production.

\section{KARGER}




\section{Materials and Methods}

\section{Participant recruitment}

Our study consecutively recruited 525 patients (276 female, 103 male) with clinically definite MS according to the criteria of McDonald and colleagues [20] from the Department of Neurology at the first affiliated hospital and the fourth affiliated hospital of Harbin Medical University in northern China. Disease severity was evaluated using the Kurtzke Expanded Disability Status Scale (EDSS).

Disease duration was defined as the time interval between disease onset and the most recent hospitalization. The progression index (PI), defined as the EDSS/ disease duration (years) , was calculated if disease duration was $\geq 5$ years. According to Lublin's criteria, 379 MS patients were categorized as having RRMS, 109 of the patients were categorized as having SPMS and 37 MS patients were categorized as having PPMS. The patients' clinical characteristics are summarized in Table 1. A total of 568 unrelated healthy blood donors (376 female, 192 male; mean age 32.5 \pm 10.7 years), matched for ethnicity, age and gender, and whose families had no history of MS or any other inflammatory-demyelinating disease, were recruited from the same geographical area in the same period as control subjects. Written informed consent was obtained from all of the enrolled participants. Ethical approval was obtained for the study from the relevant ethics committees.

\section{DNA extraction and genotyping}

Genomic DNA was extracted from whole blood samples from all of the patients and controls using the TIANamp Blood DNA Kit (Tiangen Biotech, Beijing, China) according to the manufacturer's instructions. A total of 1093 individuals were genotyped for the two single-nucleotide polymorphisms (SNPs) (rs2910164 G/C and rs57095329 A/G) using the SNaPshot technique (Applied Biosystems, Foster City, CA, USA). The PCR primers used in the SNaPshot were as follows: rs2910164F: GAACTGAATTCCATGGGTTG, rs2910164R: CACGATGACAGAGATATCCC; rs57095329F:TCATTGGGCAGCCGATAAAG, rs57095329R: AGGAAGTTCTGGTCAGGCG. Genotyping was conducted by polymerase chain reaction (PCR) according to the manufacturer's protocol as it is described previously [21]. The final data were analyzed using GeneMapper 4.1 (Applied Biosystems, Foster City, CA, USA).

\section{MiRNA extraction and real-time PCR}

To ascertain whether the genotypes of miR-146a polymorphisms (rs2910164 and rs57095329) correlated with differences in gene expression, the levels of miR-146a expression were determined by realtime PCR in peripheral blood mononuclear cells (PBMC) from 50 RRMS patients and 50 controls. PBMCs were isolated using density gradient centrifugation method with LymphoprepTM (Axis-Shield PoCAS, Oslo, Norway) as we described previously [21]. MiRNA extracted from PBMCs was performed using the miRcute miRNA Isolation Kit (Tiangen Biotech, Beijing, China) according to the manufacturer's instructions. The quantity of mature miR146a was determined by qRT-PCR using the SYBR green method as we described previously [22]. Data analysis was performed using the $2^{-\Delta \Delta \mathrm{Ct}}$ method. Values were normalized to snRNA U6.

Enzyme-linked immunosorbent assay (ELISA)

Blood samples collected for the measurement of plasma cytokines concentrations were drawn in EDTA-containing tubes. The plasma aliquots were obtained by centrifuging the blood samples at low speed. Levels of TNF- $\alpha$, IFN- $\gamma$ and IL-1 $\beta$ were measured in duplicate using Quantikine sandwich ELISA kits (R\&D Systems, Minneapolis, MN, USA) according to the manufacturer's instructions.

\section{Statistical analyses}

Statistical analyses were conducted using SPSS, version 19.0 (IBM, NY, USA) and GraphPad Prism 4.0 (GraphPad Software, Inc., San Diego, CA, USA). Allele frequencies and genotype distribution of MS patients and controls were compared using the Fisher's exact test or Chi-squared test. Deviation of the genotype or allele frequency was assessed using Hardy-Weinberg equilibrium (HWE). Analysis of variance (ANOVA) and Fisher's protected least significant difference (PLSD) were used to compare the relationships between the genotypes of miR-146a polymorphisms and the onset age or PI of MS patients. The non-parametric Mann Whitney U test was used to compare the miR-146a expression and the production of TNF- $\alpha$, IFN- $\gamma$, and IL-1 $\beta$ level among three genotypes of rs2910164 and rs57095329, respectively. The criterion for significance was set at $\mathrm{P}<0.05$ for all the tests. 
Table 1. Characteristics of MS cases and controls. Continuous data are presented as mean \pm SD. MS: multiple sclerosis, EDSS: Expanded Disability Status Scale, PI: Progression Index, PPMS: primary progressive MS, RRMS: relapsing-remitting MS, SD: standard deviation, SPMS: secondary progressive MS

\begin{tabular}{lcccc}
\hline Variables & \multicolumn{3}{c}{ MS patients $(\mathrm{n}=525)$} & controls \\
& RRMS $(\mathrm{n}=379)$ & SPMS $(\mathrm{n}=109)$ & PPMS $(\mathrm{n}=37)$ & $(\mathrm{n}=568)$ \\
\hline Mean age at onset (years) & $32.6 \pm 10.3$ & $32.1 \pm 9.6$ & $33.1 \pm 11.8$ & $32.5 \pm 10.7$ \\
Female/male & $276 / 103$ & $78 / 31$ & $27 / 10$ & $376 / 192$ \\
Mean duration & $11.5 \pm 7.8$ & $10.8 \pm 10.5$ & $11.2 \pm 9.8$ & - \\
Mean EDSS & $3.9 \pm 3.1$ & $4.0 \pm 2.6$ & $4.2 \pm 3.2$ & - \\
Mean PI & $0.42 \pm 0.32$ & $0.45 \pm 0.28$ & $0.48 \pm 0.32$ & - \\
\hline
\end{tabular}

Table 2. Frequencies of miR-146a genotypes and alleles in MS patients and controls

\begin{tabular}{|c|c|c|c|c|}
\hline Genotypes & $\begin{array}{l}\text { MS patients } \\
n=525(\%)\end{array}$ & $\begin{array}{c}\text { Controls } \\
\mathrm{n}=568(\%)\end{array}$ & OR (95\%CI) & $\begin{array}{c}P \\
\text { value }\end{array}$ \\
\hline \multicolumn{5}{|l|}{ rs2910164 } \\
\hline GG & $91(17.3)$ & $119(21.0)$ & & 0.31 \\
\hline $\mathrm{GC}$ & $252(48.0)$ & $257(45.2)$ & & \\
\hline $\mathrm{CC}$ & $182(34.7)$ & 192(33.8) & & \\
\hline Dominant model GG/GC vs CC & $343(65.3)$ & $376(66.2)$ & $0.96(0.75-1.24)$ & 0.76 \\
\hline Recessive model GG vs GC/CC & $434(82.7)$ & $449(79.0)$ & $0.79(0.58-1.07)$ & 0.13 \\
\hline G allele & $434(41.3)$ & $495(43.6)$ & 1.000 (reference) & \\
\hline C allele & $616(58.7)$ & $641(56.4)$ & $0.91(0.77-1.08)$ & 0.29 \\
\hline \multicolumn{5}{|l|}{ rs57095329 } \\
\hline $\mathrm{AA}$ & $332(63.2)$ & $337(59.3)$ & & 0.22 \\
\hline $\mathrm{AG}$ & $171(32.6)$ & $196(34.5)$ & & \\
\hline GG & $22(4.2)$ & $35(6.2)$ & & \\
\hline Dominant model AA/AG vs GG & $503(96.6)$ & $533(93.8)$ & $1.50(0.87-2.59)$ & 0.14 \\
\hline Recessive model AA vs AG/GG & 193(34.9) & $231(40.7)$ & $1.18(0.92-1.51)$ & 0.19 \\
\hline A allele & $835(80.9)$ & $870(76.6)$ & $1.000($ reference $)$ & \\
\hline G allele & $215(19.1)$ & $266(23.4)$ & $1.19(0.97-1.46)$ & 0.10 \\
\hline
\end{tabular}

\section{Results}

\section{Characteristics of the study population}

The characteristics of the MS patients and control groups are presented in Table 1. The cases and controls were matched according to age and sex.

Genotype and allele frequencies of miR-146a polymorphisms in MS patients and controls

The genotype distribution and allele frequencies of the miR-146a polymorphisms in all patients with MS and the control group are presented in Table 2. No deviation from HardyWeinberg equilibrium was observed in the genotype distributions of the MS patients and controls (data not shown). The overall genotype and allele distributions of the miR-146a rs2910164 and rs57095329 polymorphisms did not differ significantly between MS patients and controls ( $\mathrm{P}>0.05$ ) (Table 2$)$. 
Li et al.: MiR-146a Polymorphisms and Multiple Sclerosis

Table 3. Frequencies of miR-146a genotypes and alleles stratified by gender in MS patients and controls

\begin{tabular}{|c|c|c|c|c|c|c|c|c|}
\hline \multirow[b]{2}{*}{ Genotypes } & \multicolumn{2}{|c|}{ Female } & \multirow[t]{2}{*}{ OR $(95 \% \mathrm{CI})$} & \multirow{2}{*}{$\begin{array}{l}P \\
\text { value }\end{array}$} & \multicolumn{2}{|c|}{ Male } & \multirow[t]{2}{*}{ OR (95\%CI) } & \multirow{2}{*}{$\begin{array}{l}P \\
\text { value }\end{array}$} \\
\hline & $\begin{array}{l}\text { MS patients } \\
n=381(\%)\end{array}$ & $\begin{array}{l}\text { Controls } \\
n=376(\%)\end{array}$ & & & $\begin{array}{l}\text { MS patients } \\
n=144(\%)\end{array}$ & $\begin{array}{l}\text { Controls } \\
\mathrm{n}=192(\%)\end{array}$ & & \\
\hline \multicolumn{9}{|l|}{ rs2910164 } \\
\hline GG & $50(13.1)$ & $81(21.5)$ & & 0.009 & $23(16.0)$ & $40(20.8)$ & & 0.44 \\
\hline GC & $193(50.7)$ & $172(45.7)$ & & & $64(44.4)$ & $86(44.8)$ & & \\
\hline $\mathrm{CC}$ & $138(36.2)$ & $123(32.7)$ & & & $57(39.6)$ & $66(34.4)$ & & \\
\hline Dominant model GG/GC vs CC & $243(63.9)$ & $253(67.3)$ & $0.86(0.64-1.17)$ & 0.33 & $87(60.4)$ & $126(65.6)$ & $0.80(0.51-1.25)$ & 0.33 \\
\hline Recessive model GG vs GC/CC & $331(86.8)$ & $295(78.5)$ & $0.55(0.37-0.81)$ & 0.002 & $121(84.0)$ & $152(79.2)$ & $0.72(0.41-1.27)$ & 0.26 \\
\hline G allele & $293(38.5)$ & $334(44.4)$ & 1.000 (reference) & & $110(38.2)$ & $166(43.2)$ & 1.000 (reference) & \\
\hline $\mathrm{C}$ allele & $469(61.5)$ & $418(55.6)$ & $0.78(0.64-0.96)$ & 0.019 & $178(61.8)$ & $218(56.8)$ & $0.81(0.59-1.11)$ & 0.19 \\
\hline \multicolumn{9}{|l|}{ rs57095329 } \\
\hline $\mathrm{AA}$ & $246(64.6)$ & $223(59.3)$ & & 0.32 & $94(65.3)$ & $110(57.3)$ & & 0.33 \\
\hline $\mathrm{AG}$ & $117(30.7)$ & $131(34.8)$ & & & $45(31.3)$ & $74(38.5)$ & & \\
\hline GG & $18(4.7)$ & $22(5.9)$ & & & $5(3.4)$ & $8(4.2)$ & & \\
\hline Dominant model AA/AG vs GG & $363(95.3)$ & $354(94.1)$ & $1.25(0.66-2.38)$ & 0.49 & $139(96.5)$ & $184(95.8)$ & $1.21(0.39-3.78)$ & 0.74 \\
\hline Recessive model AA vs AG/GG & $135(35.4)$ & $153(40.7)$ & $1.25(0.93-1.68)$ & 0.14 & $50(34.7)$ & $82(42.7)$ & $1.40(0.90-2.19)$ & 0.14 \\
\hline A allele & $609(79.9)$ & $577(76.7)$ & 1.000 (reference) & & $233(80.9)$ & $294(76.6)$ & 1.000 (reference) & \\
\hline $\mathrm{G}$ allele & $153(20.1)$ & $175(23.3)$ & $1.21(0.95-1.54)$ & 0.13 & $55(19.1)$ & $90(23.4)$ & $1.30(0.89-1.89)$ & 0.18 \\
\hline
\end{tabular}

Table 4. Clinical characteristics according to $m i R-146 a$ genotype status. Continuous data are presented as mean \pm SD. EDSS: Expanded Disability Status Scale, PI: Progression Index

\begin{tabular}{ccccccc}
\hline Clinical features & miR-146a rs2910164 & P value & miR-146a rs57095329 & P value \\
& GG & GC/CC & & AA & AG/GG & \\
\hline No. of subjects & 91 & 434 & & 342 & 183 & \\
Most recent EDSS & $3.8 \pm 2.5$ & $4.1 \pm 2.6$ & 0.56 & $3.9 \pm 2.2$ & $4.0 \pm 1.5$ & 0.78 \\
PI & $0.41 \pm 0.22$ & $0.50 \pm 0.48$ & 0.038 & $0.46 \pm 0.36$ & $0.49 \pm 0.32$ & 0.55 \\
Age of onset, years & $31.5 \pm 10.8$ & $33.6 \pm 9.8$ & 0.81 & $32.2 \pm 9.5$ & $33.2 \pm 11.2$ & 0.25 \\
\hline
\end{tabular}

Table 5. Association between the miR-146a genotypes and alleles and MS subtypes

\begin{tabular}{|c|c|c|c|c|c|c|c|c|c|c|c|c|c|c|}
\hline & \multicolumn{7}{|c|}{ rs2910164 } & \multicolumn{7}{|c|}{ rs57095329 } \\
\hline & \multicolumn{3}{|c|}{ Genotype } & \multirow[t]{2}{*}{ P value } & \multicolumn{2}{|c|}{ Allele } & \multirow[t]{2}{*}{$P$ value } & \multicolumn{3}{|c|}{ Genotype } & \multirow[t]{2}{*}{$P$ value } & \multicolumn{2}{|c|}{ Allele } & \multirow{2}{*}{$\begin{array}{c}\mathrm{P} \\
\text { value }\end{array}$} \\
\hline & GG & GC & $\mathrm{CC}$ & & $\mathrm{G}$ & C & & $\mathrm{AA}$ & $\mathrm{AG}$ & GG & & A & G & \\
\hline Controls & $\begin{array}{l}119 \\
(21.0)\end{array}$ & $\begin{array}{l}257 \\
(45.2)\end{array}$ & $\begin{array}{l}192 \\
(33.8)\end{array}$ & & $\begin{array}{c}495 \\
(43.6)\end{array}$ & $\begin{array}{l}641 \\
(56.4)\end{array}$ & & $\begin{array}{l}337 \\
(59.3)\end{array}$ & $\begin{array}{l}196 \\
(34.5)\end{array}$ & $\begin{array}{l}35 \\
(6.2)\end{array}$ & & $\begin{array}{l}870 \\
(76.6)\end{array}$ & $\begin{array}{c}266 \\
(23.4)\end{array}$ & \\
\hline Cases & & & & & & & & & & & & & & \\
\hline RRMS & $\begin{array}{l}49 \\
(12.9)\end{array}$ & $\begin{array}{l}191 \\
(50.4)\end{array}$ & $\begin{array}{l}139 \\
(36.7)\end{array}$ & 0.007 & $\begin{array}{c}289 \\
(38.1)\end{array}$ & $\begin{array}{l}469 \\
(61.9)\end{array}$ & 0.018 & $\begin{array}{l}245 \\
(64.6)\end{array}$ & $\begin{array}{l}119 \\
(31.4)\end{array}$ & $\begin{array}{l}15 \\
(4.0)\end{array}$ & 0.15 & $\begin{array}{l}609 \\
{[80.3]}\end{array}$ & $\begin{array}{c}149 \\
(19.7)\end{array}$ & 0.053 \\
\hline SPMS & $\begin{array}{l}33 \\
(30.3)\end{array}$ & $\begin{array}{l}44 \\
(40.4)\end{array}$ & $\begin{array}{l}32 \\
(29.4)\end{array}$ & 0.10 & $\begin{array}{c}110 \\
(50.5)\end{array}$ & $\begin{array}{l}108 \\
(49.5)\end{array}$ & 0.06 & $\begin{array}{l}73 \\
(67.0)\end{array}$ & $\begin{array}{l}34 \\
(31.2)\end{array}$ & $\begin{array}{l}2 \\
(1.8)\end{array}$ & 0.12 & $\begin{array}{l}180 \\
(82.6)\end{array}$ & $\begin{array}{c}38 \\
(17.4)\end{array}$ & 0.052 \\
\hline PPMS & $\begin{array}{l}9 \\
(24.3)\end{array}$ & $\begin{array}{l}17 \\
(45.9)\end{array}$ & $\begin{array}{l}11 \\
(29.7)\end{array}$ & 0.83 & $\begin{array}{c}35 \\
(47.3)\end{array}$ & $\begin{array}{l}39 \\
(52.7)\end{array}$ & 0.53 & $\begin{array}{l}24 \\
(64.9)\end{array}$ & $\begin{array}{l}12 \\
(32.4)\end{array}$ & $\begin{array}{l}1 \\
(2.7)\end{array}$ & 0.63 & $\begin{array}{l}60 \\
(81.1)\end{array}$ & $\begin{array}{c}14 \\
(18.9)\end{array}$ & 0.37 \\
\hline
\end{tabular}

Stratification of genotype and allele frequencies of miR-146a polymorphisms in MS patients and controls by gender

When the MS patients and controls were stratified by gender, a significant difference was found in the frequency of the rs2910164 GC and CC genotype of miR-146a between female controls and female MS patients $(P=0.009)$. Additionally, a significant increase in the risk of MS was observed with a recessive model (OR=0.55, 95\% CI: $0.37-0.81, \mathrm{P}=0.002$ ) (Table 3). The miR-146a rs57095329 genotypes and alleles were not significantly associated with the risk of developing MS for either male or female individuals.

\section{Effect of miR-146a gene polymorphisms on clinical characteristics}

The general characteristics of the subjects according to their miR-146a rs2910164 and rs57095329 genotypes are shown in Table 4. The age of onset and EDSS was not significantly associated with the presence of the variant genotypes (GC/CC or AG/GG). However, our data showed that rs2910164 GC/CC carriers had a higher PI than did GG allele homozygotes (P $=0.038$ ) (Table 4). 


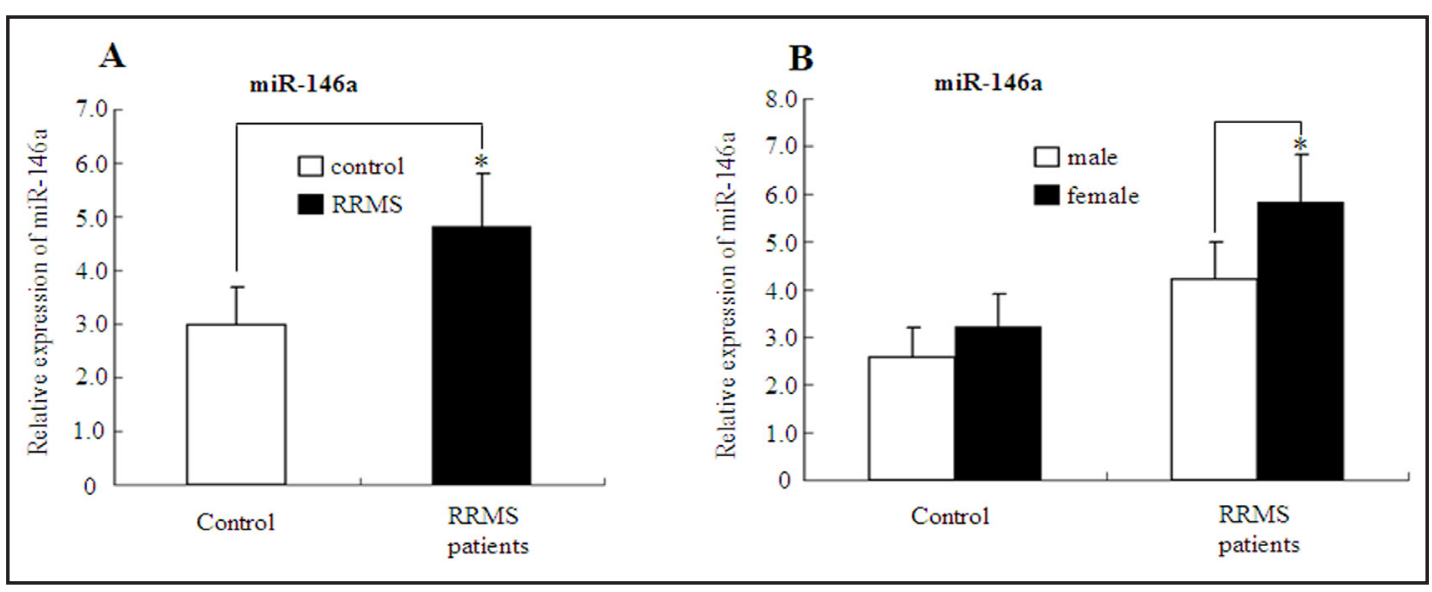

Fig. 1. (A) Mean relative miR-146a values \pm SD in PBMCs from RRMS patients (RRMS, $n=50$ ) and healthy control subjects (controls, $n=50$ ). The blank box and the black box represent the relative expression of miR$146 \mathrm{a}$ in the controls and the RRMS patients, respectively. ${ }^{*} \mathrm{P}=0.027$ when comparing relative miR-146a levels between RRMS patients and controls. (B) Mean relative miR-146a values \pm SD in PBMCs from RRMS patients (RRMS, $n=50$ ) and healthy control subjects (controls, $n=50$ ) stratified by gender. The blank box and the black box represent the relative expression of miR-146a in male and female individuals, respectively. ${ }^{*} \mathrm{P}=0.042$ when comparing relative miR-146a levels between male and female patients with RRMS.

Association of miR-146a gene polymorphisms with MS subtypes

Because MS manifests as different disease subtypes, it is important to test whether miR$146 a$ gene polymorphisms act as modifiers of the disease course. A statistically significant difference in the miR-146a rs2910164 GC and CC genotype and C allele carrier frequency between patients and controls was identified in the RRMS subgroup $(\mathrm{P}=0.007$ for genotype and $\mathrm{P}=0.018$ for allele) (Table 5). However, the number of primary, progressive MS patients was too small to draw firm conclusions. We did not find any significant association of the $m i R$ $146 a$ rs57095329 genotypes and alleles with the risk of developing MS in any MS subtypes (RRMS, SPMS or PPMS).

\section{Effect of miR-146a gene polymorphisms on miR-146a expression}

A comparative determination was performed on miR-146a expression in the PBMCs of 50 RRMS patients and 50 healthy controls. Consistent with a previous report [19], the mean value of miR-146a expression was significantly higher in the RRMS patients than in the controls ( $\mathrm{P}=0.027$ ) (Fig. 1A). We also determined the miR-146a expression levels in the patients and controls stratified by gender. An increased expression of miR-146a was detected in female patients with RRMS ( $\mathrm{P}=0.042$; Fig. 1B). However, in the normal control group, the expression of miR-146a was indistinctive in female or male individuals (Fig. 1B).

Previous studies have suggested that common GC polymorphisms might alter the free binding energy of the pre-miR-146a, which would affect the miRNA-processing efficiency [15]. To identify the effect of miR-146a polymorphisms on miR-146a expression, real-time PCR was performed to determine the expression levels of miR-146a in PBMCs of both RRMS patients and controls. The results are presented in Fig. 2. A significant increase in miR-146a expression was observed in RRMS patients who carried the mutated rs2910164 GC (P=0.028) or $\mathrm{CC}(\mathrm{P}=0.035)$ genotypes. Comparatively, a significant decrease in miR-146a expression was detected in RRMS patients who carried the mutated rs57095329 AG ( $\mathrm{P}=0.038)$ or GG $(\mathrm{P}=0.022)$ genotypes. However, no statistically significant difference in miR-146a expression was found between the population carrying the mutated allele (GC or CC for rs2910164 and AG or GG for rs57095329) and the population carrying the major GG or AA in the control subjects ( $\mathrm{P}>0.05)$ (Fig. 2). 


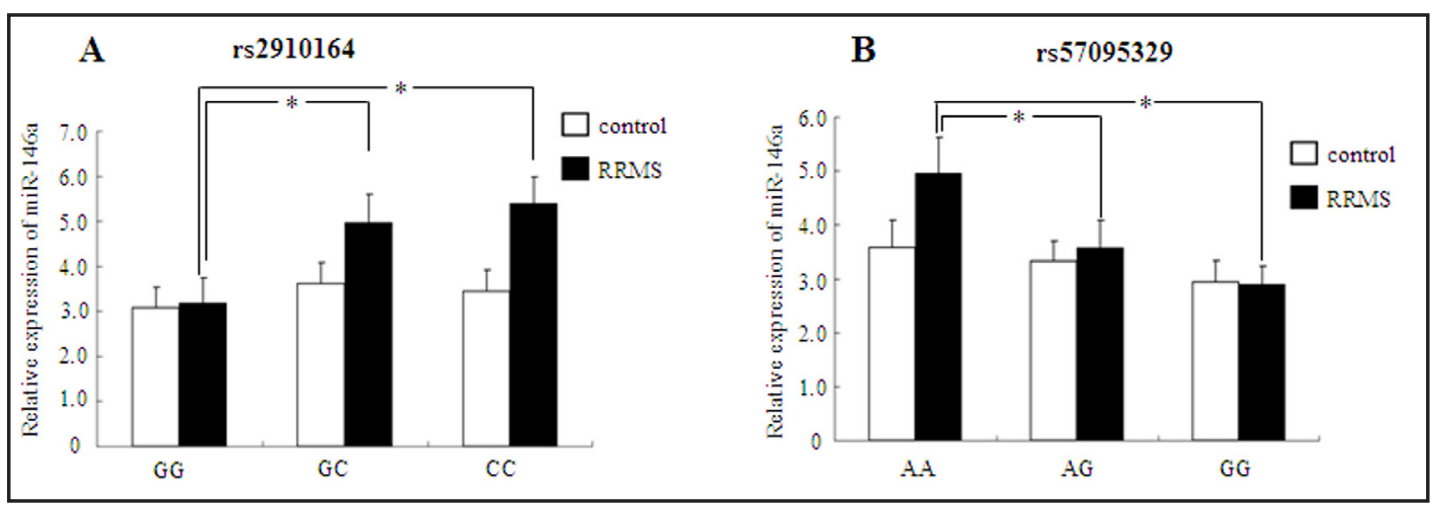

Fig. 2. Mean miR-146a values \pm SD in PBMCs isolated from the RRMS patients $(n=50)$ and controls $(n=50)$ stratified according to three different genotypes of rs2910164 and rs57095329, respectively. Real-time PCR analysis of mature miR-146a expression in PBMCs derived from the RRMS patients and healthy individuals with rs2910164 GG, GC and CC genotype and rs57095329 AA, AG and GG genotypes. Asterisk indicates $\mathrm{P}<0.05$.

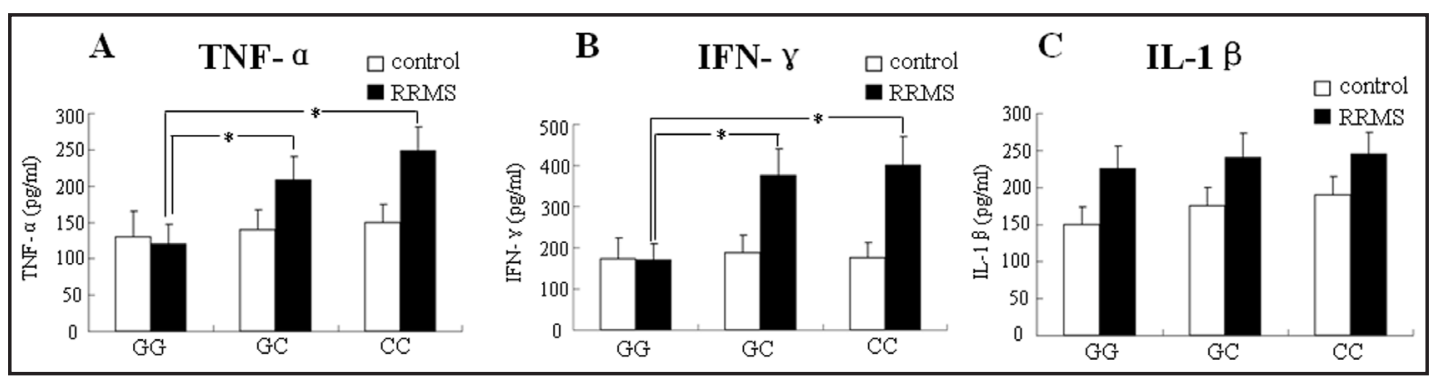

Fig. 3. Proinflammatory cytokines production with three different genotypes of rs2910164 in PBMCs. TNF- $\alpha(A), I F N-\gamma(B)$ and IL-1 $\beta(C)$ in plasma of PBMCs derived from the RRMS patients and healthy individuals with rs2910164 GG, GC and CC genotypes were measured by ELISA. Data are shown as mean \pm SD. Asterisk indicates $\mathrm{P}<0.05$.

The influence of rs2910164 polymorphism on cytokine production

Another experiment was performed to determine whether the miR-146a rs2910164 polymorphism had an effect on proinflammatory cytokine production in controls and RRMS patients. The results are shown in Fig. 3. Generally, the plasma expression levels of TNF- $\alpha$, IFN- $\gamma$, and IL- $1 \beta$ were significantly in RRMS patients than in the controls $(\mathrm{P}<0.05)$ (Fig. $3)$. Furthermore, the RRMS patients carrying the mutated GC or CC genotype presented with higher plasma TNF- $\alpha$ ( $\mathrm{P}=0.036$ for $\mathrm{GC}$ and $\mathrm{P}=0.024$ for CC) and IFN- $\gamma(\mathrm{P}=0.015$ for GC and $\mathrm{P}=0.008$ for $\mathrm{CC}$ ) levels compared to the homologous $\mathrm{GG}$ group (Fig. $3 \mathrm{~A}$ and $3 \mathrm{~B}$ ). The expression of IL-1 $\beta$ was not affected when stratified by different genotypes, in either RRMS patients or controls $(\mathrm{P}>0.05)$ (Fig. 3C).

\section{Discussion}

In this hospital-based, case-control study, we demonstrate for the first time that the rs2910164 polymorphism of $m i R-146 a$ is associated with MS in female patients and that the rs57095329 polymorphism in the promoter region of $m i R-146 a$ is not associated with MS. Further stratification revealed that the rs2910164 G>C polymorphism of miR-146a is associated with RRMS. Additionally, the miR-146a rs2910164 G>C variation modulates the expression of miR-146a and the release of TNF- $\alpha$ and IFN- $\gamma$.

MiRNAs have been recognized as important regulators in common human disorders. miR-146a is a microRNA that regulates innate immune function, inflammatory responses 
and antiviral pathways. MiR-146a may act as a negative feedback regulator of the immune response by targeting two genes, TRAF 6 and IRAK1, which are crucial for proinflammatory signaling [23]. In addition, miR-146a is important for the function of regulatory T cells, and a deficiency of this miRNA results in a defect in their suppressor function and dysregulated IFN- $\gamma$ responses [24]. Recently, Lescher et al. found that miR-146a expression was increased in EAE lesions of mice and marmosets [14]. miR-146a expression was also significantly elevated in the PBMCs of RRMS patients compared to healthy controls [19,25]. This evidence suggests that miR-146a plays an important role in MS pathogenesis. Despite these advances, miR-146a alleles that contribute to MS pathology remain undiscovered.

Polymorphisms affecting miRNA expression, maturation, or mRNA recognition may also become important determinants for an increased risk of developing MS [9]. Several studies have examined the association between the miR-146a rs2910164 polymorphism and autoimmune diseases, including RA [8], SLE [8,9] and MS [19]. Although previous studies have suggested that many autoimmune diseases share common predisposing factors, the results are inconsistent [26]. In this hospital-based, case-control study, the allele and genotype frequencies of the miR-146a polymorphisms did not differ significantly between the MS patients and healthy controls; this result is consistent with Fenoglio's previous report [19]. Interestingly, in our population, the rs 2910164 allele frequency was $56.4 \%$ for the $\mathrm{C}$ allele, which is significantly higher than the rs2910164 allele frequency $(25.0 \%)$ in Caucasians [19]. This discrepancy might result from profound ethnic differences.

However, we did find an association between the rs2910164 polymorphism and MS risk in the female subgroup of MS patients and controls. MS is universally more prevalent in females. There is evidence that gender affects the prevalence, clinical course, pathology, and prognosis of MS. It is believed that sex hormones, especially estrogen, play an important role in MS. Estrogen promotes the secretion of proinflammatory cytokines, such as IFN- $\gamma$ and IL-17 [27], either through the modification of key transcription factors in inflammation or through the regulation of miRNA expression. When splenic lymphocytes are treated with estrogen, miR-146a expression decreases [28]. This decrease may cause T-cells to become hyperactive in a chronic inflammatory autoimmune response. Other than hormones, several other factors are likely to be especially relevant in MS pathogenesis between male and female patients, including intrinsic biological differences in male and female immune systems, nervous systems, genetic and epigenetic factors, environmental exposure, and maternal microchimerism [29]. In this study, the observation that females have a higher MS prevalence than males may result from an interaction between the genotype and sex hormones during development. Gender-dependent associations have been shown in other case-control studies involving immune entities [8]. As the miR-146a gene is located on 5q33.3, one of the richest loci for genes associated with autoimmune diseases, the linkage disequilibrium between rs2910164 and SNPs near miR-146a (such as rs2431697 and rs2277920) or in other candidate genes $(A D R B 2, I L-12, I L-4, I L-13)$ should also be taken into consideration.

MiR-146a negatively modulates innate immunity through the regulation of TLR signaling and cytokine responses by targeting TRAF6 and IRAK1 [23]. In RRMS, inflammatory events play a pivotal role and occur in response to an aberrant activation of the immune system towards myelin proteins, which are supposed to act as autoantigens [19]. However, in progressive forms of MS, neurodegeneration is prominent. This neurodegeneration is demonstrated by the development of motor disabilities, cognitive deficits and brain atrophy. It is conceivable that the dysregulation of miRNAs specific to the activation and differentiation of the immune system is predominant in the acute phase of the disease. In this study, we found that the miR-146a rs2910164 GC and CC genotype and C allele are associated with RRMS. This association may occur because individuals with the rs2910164 GC and CC genotype express higher levels of miR-146a. It is likely that the rs2910164 G>C variation is linked with stronger inflammatory responses, and this hypothesis is supported by our findings that the rs2910164 GC and CC genotype is associated with higher TNF- $\alpha$ and IFN- $\gamma$ levels in RRMS patients. 
MiRNA expression analysis revealed a dysregulation of certain miRNAs in patients compared to controls. Increased miR-146a has been found in other autoimmune diseases, such as RA, SLE, and SS $[12,19]$. In the present study, we determined that miR-146a expression was significantly elevated in the PBMCs of MS patients compared to controls. This result is correlated with Fenoglio's report [19]. We also revealed that RRMS patients carrying the mutated rs2910164 GC and CC genotype have higher expression of miR146a than those with the GG genotype. However, whether the rs2910164 C allele was associated with increased or decreased miR-146a expression level is rather inconsistent. Some previous studies indicated that the rs2910164 G $>C$ variation causes mis-pairing within the miR-146a hairpin, thus leading to decreased expression of its mature form [15, 30, 31]. Nevertheless, other studies observed that the rs2910164 CC genotype was linked with increased miR-146a in breast/ovarian cancer patients [30] or coronary artery disease patients $[32,33]$. The discrepancy between these studies is intriguing, although the exact molecular mechanism of how this $\mathrm{G}>\mathrm{C}$ variation might affect the mature miRNA expression remains to be determined. Considering the effects of increased miR-146a expression on MS development, it is conceivable that individuals with C allele of rs2910164 in miR-146a may have increased miR-146a expression, which may enhance the immune and inflammatory responses, thereby contributing to increased MS risk.

The neuroinflammatory process plays a significant role in MS development, and inflammatory reactions with myeloid cells may be primordial and a possible target in MS. TNF- $\alpha$ is associated with clinical activity in RRMS and the development of progressive disease. High levels of TNF- $\alpha$ have been found in the blood and cerebrospinal fluid (CSF) of MS patients [34]. Microarray analyses of MS lesions have also demonstrated increased transcripts of genes encoding for inflammatory cytokines, particularly IL-6, IL-17, and IFN- $\gamma$ [35]. In the present study, we evaluated the effect of the rs2910164 polymorphism on plasma TNF- $\alpha$, IFN- $\gamma$ and IL- $1 \beta$ expression. Our data revealed that individuals carrying the GC and CC genotype of rs2910164 had a higher expression of TNF- $\alpha$ and IFN- $\gamma$ in RRMS patients, and the rs2910164 G>C variation had no effect on IL-1 $\beta$ production. Because miR146a negatively regulates inflammatory responses, MS patients should show decreased production of inflammatory or proinflammatory cytokines, such as TNF- $\alpha$, IFN- $\gamma$, or IL- $1 \beta$, if miR-146a expression is increased. However, we observed the inverse phenomenon in the present study. These findings imply that miR-146a might fail to accurately regulate its targets in MS due to unclear mechanisms and might lead to inefficient clearance of self-antigens and inflammation protracted in vivo, which eventually results in chronic inflammation and the activation of adaptive immune responses. Therefore, based on current knowledge, more investigations are still needed to determine the connection between miR-146a and MS.

There are several important limitations to this case-control study. First, the limited power of the present sample for the case-control study may have suppressed a true relationship due to a type II error. However, notably, the prevalence of MS in the Chinese population is lower than in the Caucasian population [1]. The mechanism that underlies this interesting ethnic difference remains unclear. Moreover, the connection between miR-146a polymorphisms and MS subtype should be interpreted with caution, because the number of SPMS and PPMS samples analyzed was quite small. Thus, these preliminary findings are not definitive and must be replicated in a larger population. Second, selection bias in the patient or control populations cannot be entirely excluded. The current results should be interpreted with caution due to the limited number of male patients. In addition, we only analyzed the effect of the miR-146a polymorphisms on miR-146a expression and proinflammatory cytokine production in RRMS patients because a positive association was found between the rs2910164 polymorphism and MS in RRMS patients and controls. Third, other risk factors in the study group, such as Epstein-Barr Virus infection, smoking and vitamin D deficiency, may have complicated the association between the miR-146a polymorphisms and MS. Finally, other functional polymorphisms may influence the expression of miR-146a or contribute to MS risk, and their combined effects must be studied to better predict the occurrence, severity, and outcome of MS. The results obtained in this study require confirmation in an 
independent study with larger populations and in different ethnic backgrounds before the conclusions can be considered definitive and useful for estimating an individual's risk for developing MS.

In conclusion, our study shows a novel, significant association between the miR-146a rs2910164 polymorphism and an increased risk of MS in female patients. Furthermore, our findings suggest that this gene polymorphism may affect the genetic predisposition to this disease through modulating the expression of mature miR-146a and influencing the release of proinflammatory cytokines in MS patients and controls. This new knowledge about miR146a may be clinically important and confirms a role for miR-146a in the pathophysiology of MS, with potentially important therapeutic implications.

\section{Disclosure Statement}

The authors have no actual or potential conflicts of interest related to this manuscript. Appropriate approval was obtained, and appropriate procedures were followed concerning human subjects.

\section{Acknowledgements}

This work was supported by funding from the National Nature Science Foundation of China (grant numbers 31171219, 81271213, 81070878, 81271214, 81300929 and 81471294); the Science and Technology Innovation Fund of Guangdong Medical College (No. STIF 201101); the Medical Scientific Research Foundation of Guangdong Province, China (A2014483 and B2014305); and the PhD Start-up Fund of Guangdong Medical College (B2012024).

\section{References}

1 Rosati G: The prevalence of multiple sclerosis in the world: An update. Neurol Sci 2001;22:117-139.

-2 Noseworthy JH, Lucchinetti C, Rodriguez M, Weinshenker BG: Multiple sclerosis. N Engl J Med 2000;343:938-952.

-3 Lassmann H, van Horssen J, Mahad D: Progressive multiple sclerosis: Pathology and pathogenesis. Nat Rev Neurol 2012;8:647-656.

4 Oksenberg JR, Barcellos LF: Multiple sclerosis genetics: Leaving no stone unturned. Genes Immun 2005;6:375-387.

5 Oksenberg JR: Decoding multiple sclerosis: An update on genomics and future directions. Expert Rev Neurother 2013;13:11-19.

6 Ramagopalan SV, Dobson R, Meier UC, Giovannoni G: Multiple sclerosis: Risk factors, prodromes, and potential causal pathways. Lancet Neurol 2010;9:727-739.

7 Hebert SS, De Strooper B: Molecular biology. MiRNAs in neurodegeneration. Science 2007;317:1179-1180.

-8 Jimenez-Morales S, Gamboa-Becerra R, Baca V, Del Rio-Navarro BE, Lopez-Ley DY, Velazquez-Cruz R, Saldana-Alvarez Y, Salas-Martinez G, Orozco L: MiR-146a polymorphism is associated with asthma but not with systemic lupus erythematosus and juvenile rheumatoid arthritis in mexican patients. Tissue Antigens 2012;80:317-321.

-9 Lofgren SE, Frostegard J, Truedsson L, Pons-Estel BA, D'Alfonso S, Witte T, Lauwerys BR, Endreffy E, Kovacs L, Vasconcelos C, Martins da Silva B, Kozyrev SV, Alarcon-Riquelme ME: Genetic association of miRNA-146a with systemic lupus erythematosus in europeans through decreased expression of the gene. Genes Immun 2012;13:268-274.

10 Yang B, Chen J, Li Y, Zhang J, Li D, Huang Z, Cai B, Li L, Shi Y, Ying B, Wang L: Association of polymorphisms in pre-miRNA with inflammatory biomarkers in rheumatoid arthritis in the chinese Han population. Hum Immunol 2012;73:101-106.

11 Pauley KM, Stewart CM, Gauna AE, Dupre LC, Kuklani R, Chan AL, Pauley BA, Reeves WH, Chan EK, Cha S: Altered miR-146a expression in sjogren's syndrome and its functional role in innate immunity. Eur J Immunol 2011;41:2029-2039.

12 Xu WD, Lu MM, Pan HF, Ye DQ: Association of microRNA-146a with autoimmune diseases. Inflammation 2012;35:1525-1529. 
13 Junker A, Krumbholz M, Eisele S, Mohan H, Augstein F, Bittner R, Lassmann H, Wekerle H, Hohlfeld R, Meinl E: Microrna profiling of multiple sclerosis lesions identifies modulators of the regulatory protein CD47. Brain 2009;132:3342-3352.

14 Lescher J, Paap F, Schultz V, Redenbach L, Scheidt U, Rosewich H, Nessler S, Fuchs E, Gartner J, Bruck W, Junker A: MicroRNA regulation in experimental autoimmune encephalomyelitis in mice and marmosets resembles regulation in human multiple sclerosis lesions. J Neuroimmunol 2012;246:27-33.

15 Jazdzewski K, Murray EL, Franssila K, Jarzab B, Schoenberg DR, de la Chapelle A: Common SNP in pre-miR146a decreases mature mir expression and predisposes to papillary thyroid carcinoma. Proc Natl Acad Sci U S A 2008;105:7269-7274.

16 Manna I, Labate A, Mumoli L, Pantusa M, Ferlazzo E, Aguglia U, Quattrone A, Gambardella A: Relationship between genetic variant in pre-microRNA-146a and genetic predisposition to temporal lobe epilepsy: A case-control study. Gene 2013;516:181-183.

17 Su XW, Yang Y, Lv ML, Li LJ, Dong W, Miao L, Gao LB, Luo HB, Yun L, Cong RJ, Liang WB, Li YB: Association between single-nucleotide polymorphisms in pre-miRNAs and the risk of asthma in a chinese population. DNA Cell Biol 2011;30:919-923.

18 Luo X, Yang W, Ye DQ, Cui H, Zhang Y, Hirankarn N, Qian X, Tang Y, Lau YL, de Vries N, Tak PP, Tsao BP, Shen $\mathrm{N}$ : A functional variant in microRNA-146a promoter modulates its expression and confers disease risk for systemic lupus erythematosus. PLoS Genet 2011;7:e1002128.

19 Fenoglio C, Cantoni C, De Riz M, Ridolfi E, Cortini F, Serpente M, Villa C, Comi C, Monaco F, Mellesi L, Valzelli S, Bresolin N, Galimberti D, Scarpini E: Expression and genetic analysis of miRNAs involved in CD4+ cell activation in patients with multiple sclerosis. Neurosci Lett 2011;504:9-12.

-20 McDonald WI, Compston A, Edan G, Goodkin D, Hartung HP, Lublin FD, McFarland HF, Paty DW, Polman CH, Reingold SC, Sandberg-Wollheim M, Sibley W, Thompson A, van den Noort S, Weinshenker BY, Wolinsky JS: Recommended diagnostic criteria for multiple sclerosis: Guidelines from the international panel on the diagnosis of multiple sclerosis. Ann Neurol 2001;50:121-127.

21 Li Y, Liao F, Yin XJ, Cui LL, Ma GD, Nong XX, Zhou HH, Chen YF, Zhao B, Li KS: An association study on ADAM10 promoter polymorphisms and atherosclerotic cerebral infarction in a chinese population. CNS Neurosci Ther 2013;19:785-794.

22 Shao Y, Li J, Cai Y, Xie Y, Ma G, Li Y, Chen Y, Liu G, Zhao B, Cui L, Li K: The functional polymorphisms of miR$146 \mathrm{a}$ are associated with susceptibility to severe sepsis in the chinese population. Mediators Inflamm 2014;2014:916202.

-23 Taganov KD, Boldin MP, Chang K-J, Baltimore D: NF-кB-dependent induction of microrna miR-146, an inhibitor targeted to signaling proteins of innate immune responses. Proc Natl Acad Sci U S A 2006;103:12481-12486.

24 Rusca N, Monticelli S: Mir-146a in immunity and disease. Mol Biol Int 2011;2011:437301.

-25 Waschbisch A, Atiya M, Linker RA, Potapov S, Schwab S, Derfuss T: Glatiramer acetate treatment normalizes deregulated microRNA expression in relapsing remitting multiple sclerosis. PLoS One 2011;6:e24604.

-26 Chen HF, Hu TT, Zheng XY, Li MQ, Luo MH, Yao YX, Chen Q, Yu SY: Association between miR-146a rs2910164 polymorphism and autoimmune diseases susceptibility: A meta-analysis. Gene 2013;521:259-264.

27 Khan D, Dai R, Karpuzoglu E, Ahmed SA: Estrogen increases, whereas IL-27 and IFN-gamma decrease, splenocyte IL-17 production in wt mice. Eur J Immunol 2010;40:2549-2556.

28 Dai R, Phillips RA, Zhang Y, Khan D, Crasta O, Ahmed SA: Suppression of LPS-induced interferon-gamma and nitric oxide in splenic lymphocytes by select estrogen-regulated micrornas: A novel mechanism of immune modulation. Blood 2008;112:4591-4597.

-29 Greer JM, McCombe PA: Role of gender in multiple sclerosis: Clinical effects and potential molecular mechanisms. J Neuroimmunol 2011;234:7-18.

30 Shen J, Ambrosone CB, DiCioccio RA, Odunsi K, Lele SB, Zhao H: A functional polymorphism in the miR146a gene and age of familial breast/ovarian cancer diagnosis. Carcinogenesis 2008;29:1963-1966.

-31 Xu B, Feng NH, Li PC, Tao J, Wu D, Zhang ZD, Tong N, Wang JF, Song NH, Zhang W: A functional polymorphism in pre-miR-146a gene is associated with prostate cancer risk and mature miR-146a expression in vivo. Prostate 2010;70:467-472.

-32 Ramkaran P, Khan S, Phulukdaree A, Moodley D, Chuturgoon AA: MiR-146a polymorphism influences levels of miR-146a, IRAK-1, and TRAF-6 in young patients with coronary artery disease. Cell Biochem Biophys 2014;68:259-266.

-33 Xiong XD, Cho M, Cai XP, Cheng J, Jing X, Cen JM, Liu X, Yang XL, Suh Y: A common variant in pre-miR-146 is associated with coronary artery disease risk and its mature miRNA expression. Mutat Res Fundam Mol Mech Mutagen 2014;761:15-20.

-34 Sharief MK, Hentges R: Association between tumor necrosis factor-alpha and disease progression in patients with multiple sclerosis. N Engl J Med 1991;325:467-472.

35 Amedei A, Prisco D, D'Elios MM: Multiple sclerosis: The role of cytokines in pathogenesis and in therapies. Int J Mol Sci 2012;13:13438-13460. 